\title{
Psychometric concerns with the 10-item Autism-Spectrum Quotient (AQ10) as a measure of trait autism in the general population
}

\author{
Emily C. Taylor ${ }^{1}$, Lucy A. Livingston ${ }^{2}$, Rachel A. Clutterbuck ${ }^{1}$ and Punit Shah ${ }^{1, \star}$ \\ ${ }^{1}$ Department of Psychology, University of Bath, Bath, BA2 7AY, United Kingdom, and ${ }^{2}$ Social, Genetic and Developmental \\ Psychiatry Centre, Institute of Psychiatry, Psychology and Neuroscience, King's College London, London, SE5 8AF, United \\ Kingdom \\ *Corresponding author: E-mail: p.shah@bath.ac.uk
}

(Received 30 September 2019; Accepted 11 December 2019)

\begin{abstract}
The 10-item Autism-Spectrum Quotient (AQ10) is a self-report questionnaire used in clinical and research settings as a diagnostic screening tool for autism in adults. The AQ10 is also increasingly being used to quantify trait autism along a unitary dimension and correlated against performance on other psychological/medical tasks. However, its psychometric properties have yet to be examined when used in this way. By analysing AQ10 data from a large non-clinical sample of adults $(n=6,595)$, we found that the AQ10 does not have a unifactorial factor structure, and instead appears to have several factors. The AQ10 also had poor internal reliability. Taken together, whilst the AQ10 has important clinical utility in screening for diagnosable autism, it may not be a psychometrically robust measure when administered in non-clinical samples from the general population. Therefore, we caution against its use as a measure of trait autism in future research.
\end{abstract}

Keywords: Autism; Psychometrics; Reliability; Screening; Questionnaire

\section{Introduction}

Autism-Spectrum Quotient (AQ) tools, widely used in psychiatry and psychology, exist in several long and short forms. The 10-item AQ (AQ10; Allison et al., 2012) is the shortest and recommended by the National Institute for Health and Care Excellence as a screening tool for autism in adults (National Institute for Health and Care Excellence, 2012) as it is sensitive to diagnosable autism. It is widely used in research and clinical practice to this end, and therefore it is crucial that the psychometric properties of this measure are robust and continually evaluated when used in new contexts and (clinical) populations. Recently, there has been an increased use of the AQ10 in large-scale studies to measure autistic traits in the general population. Specifically, overall AQ10 scores are being used to quantify the number of autistic traits/tendencies self-reported by an individual, and then correlated with their performance on other tasks (e.g., social psychological skill [Gollwitzer et al., 2019]).

\section{Objective}

In such research, it is assumed that the AQ10 measures a unitary construct, i.e., trait autism, yet its unifactorial structure was neither tested when the measure was developed, nor following its administration

\footnotetext{
(C) The Author(s) 2020. This is an Open Access article, distributed under the terms of the Creative Commons Attribution licence (http:// creativecommons.org/licenses/by/4.0/), which permits unrestricted re-use, distribution, and reproduction in any medium, provided the original work is properly cited.
} 
in recent research. There are also concerns regarding its reliability when administered in non-clinical samples (e.g., Jia et al., 2019). Therefore, we examined the AQ10's factor structure and internal reliability, supplementing the original article on its development (Allison et al., 2012) and Gollwitzer et al.'s (2019) recent research that used the AQ10 as a measure of trait autism in the general population.

\section{Methods}

Using Gollwitzer et al.'s (2019) openly accessible data (Gollwitzer, 2019) - comprising a very large sample of adults $(n=6,595)$ that had completed the AQ10 in addition to other measures - the following analyses were performed. First, confirmatory factor analysis, with maximum-likelihood estimation, tested whether a 1-factor (i.e., unifactorial) solution was a good fit to the questionnaire data. This was the critical test of whether the AQ10 is a unitary measure of trait autism. Second, parallel analysis, with oblique Promax rotation, explored if there was more than one factor present in the questionnaire data. Finally, we used several approaches to quantify the internal reliability of the AQ10. Together, we conducted this study to examine the psychometric properties of the AQ10 specifically as a measure of trait autism in the general population.

\section{Results}

The confirmatory factor analysis indices indicated that a unifactorial model poorly fit the data, with all metrics failing to meet recommended guidelines (see Hooper et al., 2008) for 'good' model fit (Table 1).

Conversely, parallel analysis showed that a four-factor solution (Table 2 and Figure 1) was more appropriate, with fit indices indicating good model fit (see Table 1).

Table 1. Factor Analysis Fit Indices for AQ10.

\begin{tabular}{llll}
\hline Fit Index & Unifactorial Model & Four-factor Model & Recommended Threshold \\
\hline Tucker Lewis Index & 0.62 & 0.95 & $\geq 0.95$ \\
\hline Root Mean Square Error of Approximation & 0.10 & 0.04 & $<0.08$ \\
\hline$\chi^{2}$ & $2334.64, p<.001$ & $115.81, p<.001$ & $p>.05$ \\
\hline
\end{tabular}

Table 2. Parallel Analysis Item Loadings of AQ10 Items.

\begin{tabular}{|c|c|c|c|c|}
\hline Autistic trait questionnaire item & Factor 1 & Factor 2 & Factor 3 & Factor 4 \\
\hline I often notice small sounds when others do not & 0.11 & -0.15 & 1.02 & -0.21 \\
\hline $\begin{array}{l}\text { I usually concentrate more on the whole picture, rather than } \\
\text { the small details }(R)\end{array}$ & 0.16 & 0.07 & 0.03 & -0.11 \\
\hline I find it easy to do more than one thing at once (R) & 0.03 & 0.62 & -0.06 & 0.04 \\
\hline $\begin{array}{l}\text { If there is an interruption, I can switch back to what I was doing } \\
\text { very quickly (R) }\end{array}$ & -0.08 & 0.82 & 0.01 & 0.05 \\
\hline $\begin{array}{l}\text { I find it easy to 'read between the lines' when someone is } \\
\text { talking to me (R) }\end{array}$ & 0.65 & 0.03 & -0.03 & 0.14 \\
\hline I know how to tell if someone listening to me is getting bored $(\mathrm{R})$ & 0.60 & -0.05 & 0.01 & 0.06 \\
\hline $\begin{array}{l}\text { When I'm reading a story, I find it difficult to work out the } \\
\text { characters' intentions }\end{array}$ & 0.04 & 0.04 & 0.00 & 0.56 \\
\hline $\begin{array}{l}\text { I like to collect information about categories of things } \\
\text { (e.g. types of car, types of bird etc.) }\end{array}$ & -0.03 & 0.03 & 0.14 & 0.07 \\
\hline $\begin{array}{l}\text { I find it easy to work out what someone is thinking or } \\
\text { feeling just by looking at their face (R) }\end{array}$ & 0.56 & -0.05 & -0.02 & 0.22 \\
\hline I find it difficult to work out people's intentions & 0.21 & -0.01 & 0.01 & 0.56 \\
\hline
\end{tabular}

Note. Table shows item loadings of ten items. (R) denotes items that are reverse scored. Item loadings greater than 0.4 are presented in bold. 


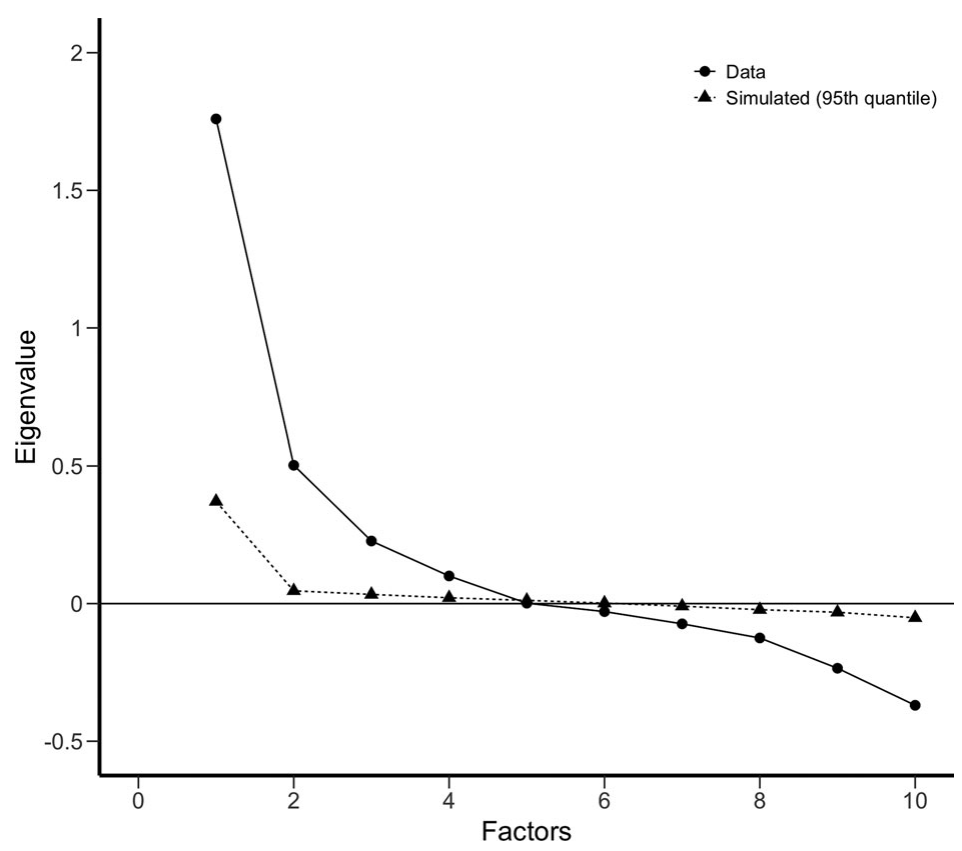

Figure 1. Scree Plot for Parallel Analysis of AQ10, which suggests that a 4-factor solution was the best fit to the data (Produced using JASP 0.11.1).

Table 3. AQ10 Reliability Statistics.

\begin{tabular}{llll}
\hline McDonald's $\omega$ & Cronbach's $\alpha$ & Gutmann's $\lambda 6$ & Average interitem correlation \\
\hline 0.577 & 0.500 & 0.579 & 0.105 \\
\hline
\end{tabular}

Finally, the AQ10 had poor internal reliability, with all metrics $<0.7$, which was unsurprising given the weak interitem correlations between the questions (Table 3).

\section{Discussions}

The results suggest that the AQ10 does not have a unifactorial structure. Rather, it appears to have multiple factors, likely because its items were drawn from 5 different subscales of the full AQ (BaronCohen et al., 2001). Therefore, its factor structure neither reflects autism conceptualised as a unitary construct, nor the dyad of social-communicative and rigid and repetitive impairments that underpin diagnosable autism (American Psychiatric Association, 2013). Given that we also found it has poor reliability, this study indicates that the AQ10 may not be a psychometrically robust measure of autism in non-clinical samples from the general population.

\section{Conclusions}

The present study is the largest psychometric analysis of the AQ10 to date. However, given the absence of socio-demographic data, it was not possible to conduct further analyses of interest (e.g., measurement invariance in males versus females). Therefore, although the AQ10 is a clinically robust screening tool for diagnosable autism, we caution against its use as a measure of trait autism in the general population until further research is conducted on its psychometric properties. We recommend that, until such research is 
conducted, AQ10 users should examine and report its psychometric properties, so this questionnaire can be evaluated and refined accordingly.

Author Contributions. ECT and PS conceived the study. ECT, RC, and PS analysed the data. ECT, LAL, and PS wrote the article.

Funding Information. ECT is support by a Whorrod Scholarship, RC by the Economic and Social Research Council, and LAL by the Medical Research Council.

Conflicts of Interest. All authors declare no conflicts of interest.

Data Availability Statement. The data used in the study are available from https://osf.io/4pd2m/?view_only=db1650b2e86240dd b7a6158a96f6abf5

\section{References}

Allison, C., Auyeung, B., \& Baron-Cohen, S. (2012). Toward brief "red flags" for autism screening: The short autism spectrum quotient and the short quantitative checklist in 1,000 cases and 3,000 controls. J Am Acad Child Adolesc Psychiatr, 51, $202-212$.

National Institute for Health and Care Excellence (2012). Autism spectrum disorder in adults: diagnosis and management (Guideline No. CG142). Retrieved from www.nice.org.uk/CG142.

Gollwitzer, A., Martel, C., McPartland, J. C., \& Bargh, J. A. (2019). Autism spectrum traits predict higher social psychological skill. Proc Natl Acad Sci USA, 116, 19245-19247.

Jia, R., Steelman, Z. R., \& Jia, H. H. (2019). Psychometric assessments of three self-report autism scales (AQ, RBQ-2A, and SQ) for General Adult Populations. J Autism Dev Disord, 49, 1949-1965.

Gollwitzer, A. (2019). "Data for autism spectrum traits predict higher social psychological skill; Study1OpenSourceNoID" https://osf.io/4pd2m/?view_only=db1650b2e86240ddb7a6158a96f6abf5, August 8, 2019.

Hooper D., Coughlan, J., \& Mullen, M. R. (2008). Structural equation modelling: Guidelines for determining model fit. Electr J Bus Res Methods, 6, 53-60.

Baron-Cohen, S., Wheelwright, S., Skinner, R., Martin, J., \& Clubley, E. (2001). The autism-spectrum quotient (AQ): Evidence from asperger syndrome/high-functioning autism, males and females, scientists and mathematicians. J Autism DevDisord, 31, 5-17.

American Psychiatric Association (2013). Diagnostic and statistical manual of mental disorders: DSM-5 (5 $\left.{ }^{\text {th }}\right)$. Washington, D.C: American Psychiatric Association.

Cite this article: Taylor EC, Livingston LA, Clutterbuck RA, Shah P (2020). Psychometric concerns with the 10-item Autism-Spectrum Quotient (AQ10) as a measure of trait autism in the general population. Experimental Results, 1, e3, 1-6. https://doi.org/10.1017/exp.2019.3 


\section{Peer Reviews}

\section{Reviewing editor: Dr. Christine Payne}

NYU Langone Medical Center, Child and Adolescent Psychiatry, New York, New York, United States, 10016-4576

This article has been accepted because it is deemed to be scientifically sound, has the correct controls, has appropriate methodology and is statistically valid, and met required revisions.

\section{doi:10.1017/exp.2019.3.pr1}

Review 1: Psychometric concerns with the 10-item Autism-Spectrum Quotient (AQ10) as a measure of trait autism in the general population

Reviewer: Katy Unwin (D)

Cardiff University

Date of review: 11 October 2019

Published online:

Conflict of interest statement. Reviewer declares none

Comments to the Author: This is a valuable contribution to the literature. It would benefit from being more succinct in the introduction and discussion, and referencing previous reliability and validity findings. Could include comment on 4 -factor structure appearing meaningful, i.e. Factor 4 items 'Theory of Mind' sub-scale.

\section{Score Card}

Presentation

4.1

Is the article written in clear and proper English? (30\%)

Is the data presented in the most useful manner? (40\%)

Does the paper cite relevant and related articles appropriately? (30\%)

\section{Context}

Does the title suitably represent the article? (25\%)

Does the abstract correctly embody the content of the article? (25\%)

Does the introduction give appropriate context? (25\%)

Is the objective of the experiment clearly defined? (25\%)

Are the limitations of the experiment as well as the contributions of the experiment clearly outlined? (20\%) 
Review 2: Psychometric concerns with the 10-item Autism-Spectrum Quotient (AQ10) as a measure of trait autism in the general population

Reviewer: Dr. Steven Stagg (D)

Anglia Ruskin University, Psychology and Sport Science, Chelmsford, United Kingdom of Great Britain and Northern Ireland, CM1 1SQ

Date of review: 26 October 2019

Published online:

Conflict of interest statement. Reviewer declares none

Comments to the Author: The paper presents an interesting analysis of the psychometric properties of the $A Q$. It has a valuable contribution to make to researchers who may use the AQ10 as a measure of autistic traits within non-autistic populations. All sections are written very concisely without losing clarity, and the paper is written in an accessible style.

\section{Score Card}

Presentation

5.0

Is the article written in clear and proper English? (30\%)

Is the data presented in the most useful manner? (40\%)

Does the paper cite relevant and related articles appropriately? (30\%)

\section{Context}

5.0

Does the title suitably represent the article? (25\%)

Does the abstract correctly embody the content of the article? (25\%)

Does the introduction give appropriate context? (25\%)

Is the objective of the experiment clearly defined? (25\%)

Are the limitations of the experiment as well as the contributions of the experiment clearly outlined? $(20 \%)$ 